\title{
Heilgymnastik als Frauenberuf
}

1903 eröffnete in Kiel der deutsche Arzt Johann Hermann Lubinus eine heilgymnastische Lehranstalt für Frauen, die er bis 1926 in den theoretischen Fächern selbst unterrichtete. Die Entscheidung Lubinus', ausschließlich Frauen auszubilden, machten den Beruf des Heilgymnasten damit zu einem Frauenberuf. Noch heute werden in der Bundesrepublik Deutschland, anders als in anderen europäischen Ländern, überwiegend Frauen als Krankengymnast/in ausgebildet. Lubinus ist mit der Gründung dieser heilgymnastischen Lehranstalt als Vater der deutschen Krankengymnastik und Initiator der Krankengymnastikschulen in Deutschland zu betrachten.

Als Folge der verheerenden Weltkriege wandten sich vermehrt traumatisierte und an Gliedmaßen verletzte Patienten hilfesuchend an Krankengymnasten. Auch die Behandlung der vielen Betroffenen, die an Kinderlähmung erkrankt waren, fiel nun in den Aufgabenbereich des Krankengymnasten. 1917 erschien Lubinus’ „Lehrbuch der Medicinischen Gymnastik“. In dem Vorwort beschrieb er die bereits errungenen Erfolge der medizinischen Gymnastik in der Traumatologie, sah aber noch Chancen für die medizinische Gymnastik in der Neurologie und inneren Medizin: „Die Heilgymnastik erfreut sich ärztlicherseits auf dem Gebiete der Inneren und Nervenkrankheiten immer noch nicht der Wertschätzung, die diesem Heilfaktor mit Recht gebührt. Auf chirurgischem und orthopädischem Gebiet dagegen hat sie ihr Ausbreitungsgebiet in den letzten Jahrzenten wesentlich zu vergrößern gewußt und besonders in diesem großen Weltkriege zum Segen für unsere verwundeten Krieger sich herrlich bewährt". Nach Lubinus verliert sich der krankengymnastische Beruf durch u. a. die sehr hohen Schulgebühren, die sehr niedrige Akzeptanz von Ärzten, die schlechte Bezahlung sowie zu wenig Stellenangebote (Langohr o. J.; Scheel 2012). 